\title{
Fiber optic acoustic pressure sensor with high dynamic range and low noise
}

\author{
Markus J. Schmid ${ }^{1}$, Dr.-Ing. Mathias S. Müller ${ }^{1}$, Bernd A. Kuhnle ${ }^{1}$, \\ Dr.rer.nat. Michael W. Bauer ${ }^{2}$, Reinhard Pongratz ${ }^{2}$, \\ Dr.-Ing. Andree Altmikus ${ }^{3}$ \\ ${ }^{1}$ fos $4 X \mathrm{GmbH}$, Thalkirchner Str. 210, 81371 Munich, Germany, www.fos4X.de \\ Markus.Schmid@fos4X.de \\ ${ }^{2}$ AIRBUS GROUP Innovations, Willy-Messerschmitt-Str. 1, 82024 Taufkirchen, Germany \\ ${ }^{3}$ Wobben Research and Development GmbH, Borsigstr. 26, 26607 Aurich, Germany
}

\begin{abstract}
:
Fiber optic sensors for temperature, strain and acceleration are well established and widely available. Here, we present a new all fiber optic acoustic pressure sensor with low noise and high dynamic range and high bandwidth. The sensor is capable of operating in very harsh environment including humidity, water, temporal overpressure, electro-magnetic noise, lightning and environmental impact such as heavy rain and thus applicable in field. Data measured during a laboratory test campaign in a flow channel in cooperation with AIRBUS GROUP Innovations are presented and compared to existing electrical microphones.
\end{abstract}

Key words: aeroacoustics pressure sensor, fiber optic, static and dynamic pressure sensing, rugged

\section{Motivation}

Objects moving through air or overflowed by air generate sound because of unsteady pressures around them. [1] By measuring the acoustic emission in wind tunnels or in the field the sources can be detected and optimized. Furthermore microphones in airfoils enable acoustic pitch control by measuring the transition of boundary layer from laminar to turbulent flow. [2]

State of the art microphones have disadvantages, which avoid the said applications, like weak and therefore drawn back membrane, unsteady spectral characteristics and resonances, electro-magnetic interference, and corrosion.

\section{Introduction}

Here we present the fiber optic pure glass static and acoustic pressure sensor (see Fig. 1). The fos $4 \mathrm{X} \mathrm{GmbH}$ developed the sensor together with Wobben Research and Development $\mathrm{GmbH}$, the research division of ENERCON $\mathrm{GmbH}$, for static and dynamic pressure sensing in rotor blades of wind energy converters.

The sensor with dimensions of $2 \times 3 \times 10 \mathrm{~mm}^{3}$ has a glass membrane with $1.7 \mathrm{~mm}$ in diameter. Due to the small size the resonance frequency is above $250 \mathrm{kHz}$. The sensor is capable of measuring static and acoustic signals with a linear transfer characteristic up to $40 \mathrm{kPa}$, and tested for nondestructive overpressures of 10 bar. With the flat design the optic microphone can be integrated in surfaces with minimal aerodynamic disturbance to the test specimen. The open membrane at the surface of the sensor enables acoustic measurements without spectral characterization, because of constant frequency response to around $80 \%$ of the resonant frequency of $250 \mathrm{kHz}$.

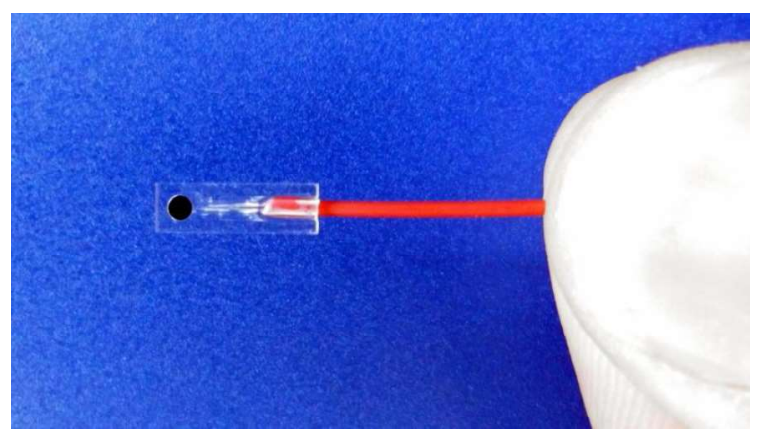

Fig. 1. Fiber optic pressure sensor fos4Pressure by fos $4 X$ for static and dynamic pressure sensing.

The fiber microphone is a passive optic sensor without any conductive material, and therefore robust against water, humidity, and corrosion. Due to the absence of conductive material the sensor is inherent immune to electro-magnetic interference, and furthermore to lightning strikes. 
The fiber optic sensor is connected to the measurement device via a telecommunication single mode fiber with very low attenuation, so large distances up to several hundred meters between sensor and measurement device can be realized.

\section{Test setup}

In cooperation with AIRBUS GROUP Innovations fos $4 \mathrm{X}$ executed a verification test of the fiber optic pressure sensor in an AIRBUS flow channel. The flow channel is capable of laminar air flow up to $212 \mathrm{~m} / \mathrm{s}$.

For the test we integrated two fiber optic pressure sensors (fos4Pressure-1 and -2) by fos $4 \mathrm{X}$ and one electric reference microphone (electric microphone) by Brüel\&Kjær in the surface of the test nozzle's outlet (see figure 2).

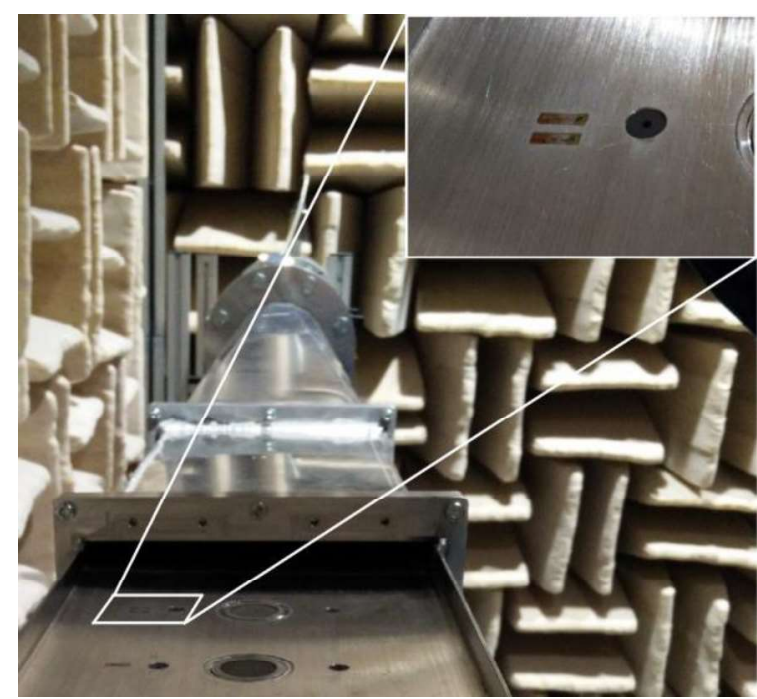

Fig. 2. Flow channel by AIRBUS GROUP Innovations with integrated fiber optic pressure sensors fos4Pressure-1 and fos4Pressure-2 (square objects in detail view), and the electric microphone (round object in detail view).

Before the measurement we calibrated both sensor types using an acoustic calibrator with a single tone at frequency $1 \mathrm{kHz}$ and amplitude $114 \mathrm{~dB}(\mathrm{SPL})$. Figure 3 shows the power spectral density of the fiber optic pressure sensor and the electric microphone measuring the calibration signal including the optic sensor noise floor of $0.001 \mathrm{~Pa}^{2} / \mathrm{Hz}$ and therefore the broadened spectrum compared to the electric microphone. The noise floor of the electric microphone is lower in comparison. Therefore the widening of the spectrum and the noise floor is not illustrated in figure 3 .

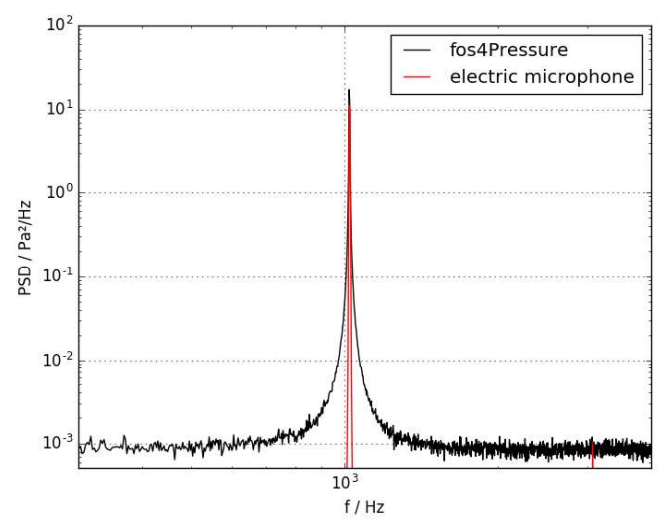

Fig. 3. Power spectral density of the fiber optic pressure sensor fos4Pressure and the electric microphone of the calibration signal $1 \mathrm{kHz}$, $114 \mathrm{~dB}(S P L)$.

In reference to the electric microphone the fiber optic pressure sensor exhibits a deviation of $1 \mathrm{~Hz}$ in frequency and $0.2 \mathrm{~dB}(\mathrm{SPL})$ in amplitude.

The integration of microphones in surfaces can disturb the air flow of the tunnel and therefore generate undesirable acoustic emission. For the test we applied a high density wire mesh over all sensors to reduce the parasitic flow noise. To compare the influence of the surface integrated microphones and the wire mesh we executed two similar runs with and without wire mesh.

\section{Results}

Figure 4 illustrates the power spectral density of electric and fiber optic microphone at flow speed $46 \mathrm{~m} / \mathrm{s}$. We executed two runs at that flow speed, one with and one without the high density wire mesh.

The electric microphone - compared to the fiber optic sensor - is strongly influenced by the wire mesh at frequencies greater $2 \mathrm{kHz}$.

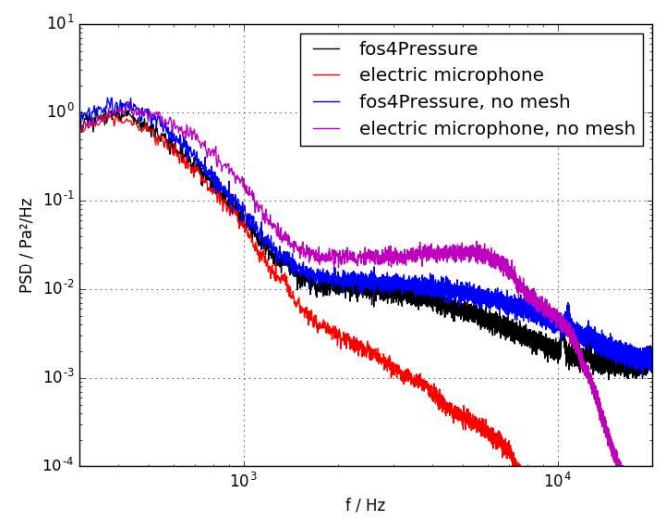

Fig. 4. Power spectral density of the fiber optic pressure sensor fos4Pressure-1 and the electric microphone at wind speed $46 \mathrm{~m} / \mathrm{s}$. The plot shows 
two runs; with and without high density wire mesh over the sensors.

In figure 5 the power spectral density of electric and fiber optic microphone at flow speed $92 \mathrm{~m} / \mathrm{s}$ is presented for two runs with and without high density wire mesh.

At around $10 \mathrm{kHz}$ a dominant parasitic acoustic resonance appears for the electric microphone, which is reduced by the applied mesh. The optic sensor shows no significant influence by the mesh.

The colored noise between $1 \mathrm{kHz}$ and $2 \mathrm{kHz}$ appears on both sensors and independent of the mesh, and therefore can be interpreted as background noise of the flow channel due to aerodynamic obstacles.

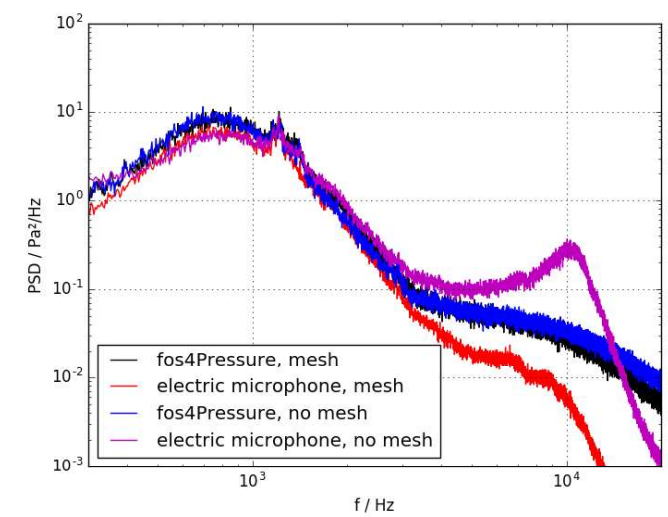

Fig. 5. Power spectral density of the fiber optic pressure sensor fos4Pressure-1 and the electric microphone at wind speed $92 \mathrm{~m} / \mathrm{s}$. The plot shows two runs; with and without high density wire mesh over the sensors.

As further benchmark figure 6 illustrates the power spectral density of the two fiber optic pressure sensors fos4Pressure-1 and -2 at $92 \mathrm{~m} / \mathrm{s}$ flow speed without high density wire mesh. The geometrical distance of the two optic sensors is about $6 \mathrm{~mm}$, therefore sound waves up to approximately $10 \mathrm{kHz}$ appear as pressure equilibrium on both sensors. The illustrated power density plots of the neighboring optic pressure sensors show the expected high spectral correlation.

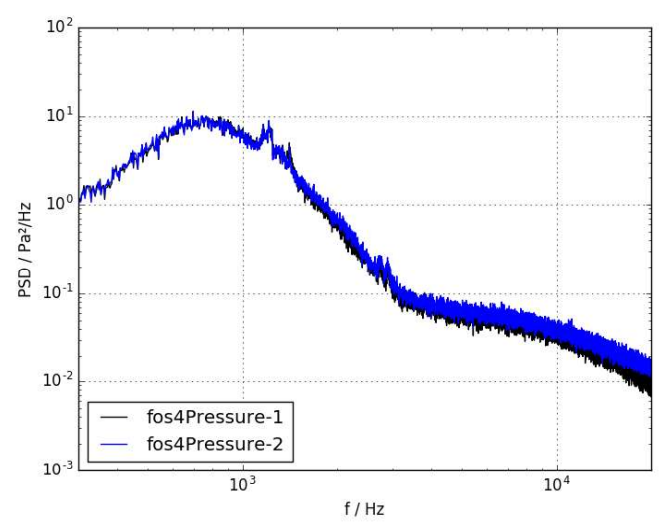

Fig. 7. Power spectral density of the fiber optic pressure sensors fos4Pressure-1 and fos4Pressure2 at wind speed $92 \mathrm{~m} / \mathrm{s}$ without high density wire mesh.

\section{Conclusion}

We demonstrated a fiber optic pressure sensor with high dynamic and frequency range. Because of all glass construction the sensor can be installed in harsh environments including humidity, water, temporary overpressure, and electro-magnetic noise. Due to the small dimension the sensor is capable of surface integration. Furthermore the real surface membrane enables measurements of noisy boundary layers with minimal aerodynamic and aeroacoustics disturbance.

In cooperation with AIRBUS GROUP Innovations we applied a benchmark test with conventional electric microphones in a flow channel.

The fiber optic pressure sensor shows good performance compared to conventional microphones. Furthermore, due to the open surface membrane of the optic sensor, no parasitic acoustic disturbances occur.

\section{References}

[1] T. J. Müller, Aeroacoustic measurements, Springer (2002); doi: 10.1007/978-3-662-05058-3

[2] W. Nitsche, Strömungsmesstchnik, Springer (1994); doi: 10.1007/978-3-662-10111-7 